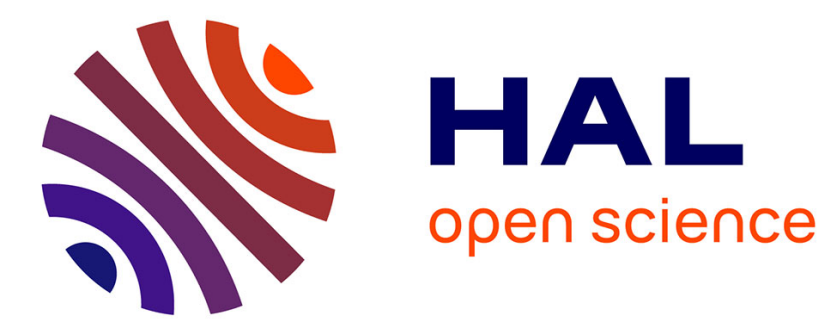

\title{
Live seeding: Performance bounds of seeders for P2P live streaming
}

Fabien Mathieu

\section{To cite this version:}

Fabien Mathieu. Live seeding: Performance bounds of seeders for P2P live streaming. P2P'11

- International Conference on Peer-to-Peer Computing, Aug 2011, Kyoto, Japan. pp.172 -181, 10.1109/P2P.2011.6038733 . hal-00667457

\section{HAL Id: hal-00667457 \\ https://hal.inria.fr/hal-00667457}

Submitted on 7 Feb 2012

HAL is a multi-disciplinary open access archive for the deposit and dissemination of scientific research documents, whether they are published or not. The documents may come from teaching and research institutions in France or abroad, or from public or private research centers.
L'archive ouverte pluridisciplinaire HAL, est destinée au dépôt et à la diffusion de documents scientifiques de niveau recherche, publiés ou non, émanant des établissements d'enseignement et de recherche français ou étrangers, des laboratoires publics ou privés. 


\title{
Live Seeding: Performance Bounds of Seeders for P2P Live Streaming
}

\author{
Fabien Mathieu \\ INRIA, University Paris 7, France \\ Email: fabien.mathieu@inria.fr
}

\begin{abstract}
Seeders (peers that do not request anything but contribute to the system) allow to leverage the capacities of a P2P system. While seeding is a natural idea for filesharing or Videoon-Demand applications, it seems somehow counter-intuitive in the context of live streaming. This paper aims at describing the feasibility and performance of P2P live seeding.

After a formal definition of "live seeding" and efficiency, we consider the theoretical performance of systems where the overhead is neglected. We then propose a realistic overhead model and extend the results for this model. The performance of a single seeder and a set of seeders are considered, as it is not always possible to perfectly aggregate individual efficiencies.

In details, we provide an explicit upper bound of seeders' achievable efficiency in a P2P system with linear overhead. We also propose and study two simple mechanisms that allow to deploy a live seeding architecture while handling seeders aggregation, providing near-optimal seeding.
\end{abstract}

\section{INTRODUCTION}

Upload bandwidth is one of the main bottleneck in peerto-peer (P2P) content distribution, which relies on the upload capacity of its participants to achieve its purpose. The upload resource is all the more critical since most todays high speed Internet access are asymmetric DSLs connections that are not designed to handle P2P traffic and offer relatively low upload capacity, with typical uplink/downlink ratios between $1 / 4$ and $1 / 20$. The democratization of very high speed, symmetric, Internet access like FTTH is expected to improve the upload capacity of P2P systems but, on the other hand, the evolution of content quality standards makes the requirements in terms of content size and rate higher and higher: earlier video feeds on the Internet were low quality, requiring streamrates of a few hundred kbps, whereas HDTV implies rates of up to 20 Mbps, possibly more with the upcoming of 3D video content. It is therefore likely possible that upload will remain a major bottleneck of tomorrow's P2P content distribution.

\section{A. Motivation}

In order to increase the available resources, a standard P2P technique is to leverage the capacity of the system by using seeders, i.e. peers that contribute to the system but are (currently) not needing anything. Using seeders is quite natural for filesharing (FS) or Video-on-Demand (VoD): after a peer has downloaded a given file or video, it becomes a natural seeder for that content. However, seeding is counter-intuitive for live streaming (LS) systems: as "live" content is created on the fly, it cannot be pro-actively possessed by peers. Therefore, for a peer to act as a seeder, it has to receive at least a part of the corresponding content, which it does not want to watch by definition.

\section{B. Scope and contribution}

The goal of this paper is to describe the feasibility and performance one can expect from $\mathrm{P} 2 \mathrm{P}$ live seeding from a bandwidth budget perspective. This generic theoretical framework can be used to derive simple dimensioning rules and recommendations for the design of $\mathrm{P} 2 \mathrm{P}$ live streaming with seeders.

In details, we analyze the seeders' efficiency, which is the goodput (i.e. the useful throughput) they add to the system, compared to their upload capacity. We provide explicit, tight, upper bounds for efficiency, taking the overhead explicitly into account. We also address the aggregation issues that come from using several seeders. We give conditions and simple diffusion schemes that allow to nearly achieve the theoretical bounds, and provide a few simple examples that illustrate the potential of our findings.

Remark: We made the deliberate choice to focus on a single scenario (live streaming) and a single type of peer (seeders). The reason was to get a clean framework for investigating theoretical performance, especially with regards to the overhead modeling aspects. This does not preclude of possible extensions of the approach presented here to other use cases.

\section{Roadmap}

The next Section introduces the models we use throughout the article. The related work with respect to P2P bandwidth dimensioning is briefly exposed in Section III. Section IV proposes a formal definition of seeders' efficiency. Section V gives a preliminary study of efficiency for two overhead-free models. It serves as a starting point for the main results of this paper, which derive the efficiency of seeders in a model with explicit overhead (Section VI). Applications of the results are discussed in Section VII. Section VIII concludes.

\section{MODEL}

We consider a live content that needs to be streamed to a set of users at a constant rate $r$. The delivery is handled by a P2P live streaming system. In contrast to FS or VoD, LS content cannot be prefetched. A play-out buffer may tolerate some jitter, but the live constraints usually limit the size of that buffer to less than a few seconds. It is therefore conservative, 
yet realistic, to assume that LS content must received at exactly the rate $r$ during the whole watching experience. To compare with, FS content usually requires no minimal rate, while VoD content may be prefetched at a rate greater than $r$.

\section{A. $C / S / L$ systems}

We classify the nodes of the system into three categories:

- Central servers are in charge of injecting initial copies of the stream into the system. We assume they have a cumulated bandwidth capacity that allows to inject $N_{C}$ copies of the stream, with $N_{C} \geq 1$.

- Leechers are peers that want to watch the live content.

- Seeders ${ }^{1}$ are peers that do not want to watch the live content, but can provide bandwidth to the system.

In this model, the main difference between servers and seeders is that servers have implicit access to the feed. For instance, nodes of a content delivery system should be classified as servers if you do not consider how the feed is transmitted to them in the bandwidth budget, or as seeders otherwise.

Remark: we do not focus on the way seeders could be enforced in a real live streaming system. However, most of the ideas used in FS or VoD systems should apply to live streaming. For instance:

- Some idle peers may remain connected to the system.

- In a multi-channel system, leechers from an overprovisioned channel may act as seeders for another channel that lacks resources.

- A share-ratio policy be used: peers that do not offer enough instant bandwidth may have to act as seeders for a while in order to "pay" their bandwidth debt. That kind of policy can be enforced through penalties (no service guarantee, reduced catalog) and rewards (higher QoS, access to premium content).

- In the case of CDNs or ISP-managed networks, nodes may be deployed by a provider (of content or network) to enhance the system performance.

We denote by $C, L$ and $S$ the sets of servers, leechers and seeders respectively. The number of leechers (resp. seeders) is denoted by $N_{L}$ (resp. $N_{S}$ ). Every peer $p$ in $L$ or $S$ has an upload capacity $u_{p} \geq 0$ devoted to the service. We assume that the download capacity is always sufficient to support the content rate $r$ and a possible overhead. $U_{X}$ and $\bar{u}_{X}$ are respectively the total and average upload bandwidths of set $X$ $\left(\bar{u}_{X}=\frac{U_{X}}{N_{X}}\right)$.

Note that the bandwidth distribution of the seeders may differ from the one of the leechers. For instance, if seeders are former leechers forced to remain because of some share-ratio policy, low bandwidth peers will have to seed longer [2], so the average seeders' bandwidth will be lower than the leecher's one. One the other hand, seeders deployed by some content or network provider are expected to deliver higher bandwidths.

A diffusion scheme for the system is a policy that describes how the content is distributed. We assume here static diffusion schemes: between any two peers (or servers) $p$ and $q$, the

\footnotetext{
${ }^{1}$ The terms leecher and seeder come from the BitTorrent vocabulary [1].
}

scheme gives a stream of goodput $0 \leq r_{p, q} \leq r$ that is sent from $p$ to $q$. If $0<r_{p, q}<r, r_{p, q}$ is called a substream. For convenience, we consider that the substreams received by a given peer are non-overlapping, so a peer $p$ receives an input of rate

$$
i_{p}=\sum_{q \in\{L, S, C\}} r_{q, p}
$$

Remark: overlapping substreams can always be seen as non-overlapping ones: if $r_{p, q}$ and $r_{s, q}$ are overlapping, with redundant data of rate $r_{p \cap s, q}$, we just have to consider $\tilde{r}_{p, q}:=$ $r_{p, q}-r_{p \cap s, q}$ and see a rate $r_{p \cap s, q}$ from $p$ to $q$ as overhead. Choosing $p$ or $s$ as sender of the redundant data is arbitrary.

Servers apart, a node cannot send something it doesn't possess, so a diffusion scheme verifies the condition

$$
\forall p, q \in\{L, S\}, r_{p, q} \leq i_{p} .
$$

A scheme is a solution of the live diffusion if it ensures that all leechers can view the content, i.e.

$$
\forall p \in\{L\}, i_{p}=r .
$$

\section{B. Connectivity}

In this work, we use an explicit linear overhead to account for connectivity constraints. We also propose two simpler models that will serve for didactic purposes: perfect systems and limited fanout systems.

1) Perfect systems: In perfect systems, peers can arbitrarily use the upload capacity devoted to the service at no cost [3]. In particular, a perfect system possesses the following properties:

- No overhead: all the bandwidth capacity can be used to effective data transfer (goodput);

- Unlimited fanout: one peer can send data to an arbitrary numbers of other peers simultaneously;

- Stream continuity: the live stream can be divided into arbitrary small substreams of constant rate.

2) Limited fanout: As we will see in Section V, optimizing perfect systems often leads to full mesh solutions, which are not realistic. A first idea to obtain more viable solutions, without explicitly considering the overhead, is to assume that the number of non-null substreams $r_{p, q}$ is limited: each peer $p$ has a limit $c_{p}$ on the number of outgoing connections it can sustain. This limited fanout implicitly acknowledges that managing a connection has a cost. Perfect systems correspond to the extreme case $c_{p}=\infty$, for all $p \in\{L, S\}$.

3) Explicit linear overhead: In order to increase the realism of our model, we propose to assume that the overhead is linear: the actual bandwidth used to transmit at a rate $e$ from one peer to another is $(1+a) e+b$, for some constants $a, b \geq 0$. $a$ is the proportional cost and $b$ the additive cost. For simplicity, we consider that all the overhead cost is supported by the sender (this assumption will be discussed in VI-B5).

The motivation for this model is that most existing sources of overhead are, at least in a rough approximation, proportional or additive:

- Periodic signaling messages (keep-alive, overlay maintenance) are additive; 
- In chunk-based systems, the stream is split into atomic units of data (the chunks) that are distributed independently. The signaling per transmitted chunk is expected to be constant, inducing a proportional overhead if chunks are homogeneous;

- The cost for initiating a connection, averaged over the lifetime of that connection, can be considered as additive;

- Some randomized diffusion scheme can have a non-null probability to send useless data, because it is outdated or redundant [4]. This can be seen as proportional overhead.

With this model, a peer of bandwidth $u$ maintaining $c$ outgoing connections has a useful output limited to $\frac{u-b c}{1+a}$. For $b>0,\left\lfloor\frac{u}{b}\right\rfloor$ is the maximal sustainable fanout. For $b=0$, the model is indeed equivalent to perfect systems, except that all bandwidth capacities have to be normalized by $\frac{1}{1+a}$.

The notation used is summarized in Table I.

TABLE I

TABLE OF NOTATION

\begin{tabular}{|c|l|}
\hline$r$ & Streamrate of the content (constant) \\
$u_{p}$ & Available upload bandwidth of peer $p$ \\
$U_{X} / \bar{u}_{X}$ & Total/average upload capacity of population $X$ \\
\hline$N_{X}$ & Number of nodes in $X$ \\
$N_{C}$ & Normalized capacity of servers $\left(U_{C}=N_{C} R\right)$ \\
$i_{p}$ & Input rate of node $p$ \\
$r_{p, q}$ & Substream from $p$ to $q$ \\
$\eta_{d}(X)$ & Efficiency of set $X$ in diffusion scheme $d$ \\
$c_{p}$ & Fanout of peer $p$ \\
$a$ & Proportional cost of a connection \\
$b$ & Constant cost of a connection \\
$R:=(1+a) r+b$ & Bandwidth consummed by goodput $r$ \\
\hline
\end{tabular}

\section{RELATED WORK}

Understanding the bandwidth dimensioning is a crucial question in P2P systems, as upload bandwidth is a scarce resource. The bandwidth conservation law [2] tells that, if all available bandwidth resources can be used to useful content transfer, then the condition for a live streaming system to admit a solution is

$$
\alpha_{L}+\beta \alpha_{S}+\frac{N_{C}}{N_{L}} \geq 1, \text { with }\left\{\begin{array}{l}
\alpha_{X}=\frac{\bar{u}_{X}}{r}, \\
\beta=\frac{N_{S}}{N_{L}} .
\end{array}\right.
$$

In reality, not all bandwidth can be used all the time. Overhead issues aside, several phenomena can prevent from using all available bandwidth. For instance, a peer may have nothing to give at a given time; or some bandwidth may be required for other purposes than feeding the leechers. This can be modeled by an efficiency parameter. Taking efficiency into account, equation (4) becomes

$$
\eta(L) \alpha_{L}+\eta(S) \beta \alpha_{S}+\eta(C) \frac{N_{C}}{N_{L}} \geq 1,
$$

where $\eta(X)$ is the efficiency of set $X$.

Efficiency was introduced by Qiu and Srikant [5] for BitTorrent-like filesharing systems [1]. Its role was to quantify the fact that leechers may lack the content required by others, preventing them to upload at full bandwidth capacity.
In the case of standard peer-assisted live streaming, with no seeders $(S=\emptyset)$, Liu et al. have shown that one can reach $\eta(L)=\eta(C)=1$ for perfect and limited fanout systems. In other words, for overhead-free systems, a perfect use of the available bandwidth can be achieved [3].

\section{DEFINING SEEDERS' EFFICIENCIES}

We propose to extend the concept of efficiency to seeders as follows: in a given diffusion scheme $d$, the efficiency $\eta_{d}(s)$ of a seeder $s$ is the ratio between the data bandwidth it adds to the system and its upload bandwidth $u_{s}$. Note that the input rate $i_{s}$ received by $s$ is "wasted" in the bandwidth budget, as $s$ does not need to receive any of the content. We say that $s$ "removes" $i_{s}$ from the pool of useful resources, in a matter of speaking ${ }^{2}$. Formally, if, in a scheme $d, s$ transmits at rates $r_{s, p_{1}}, \ldots, r_{s, p_{c}}$ to $c$ other peers (cf Figure 1), its efficiency is

$$
\eta_{d}(s):=\frac{\sum_{k=1}^{c} r_{s, p_{k}}-i_{s}}{u_{s}} .
$$

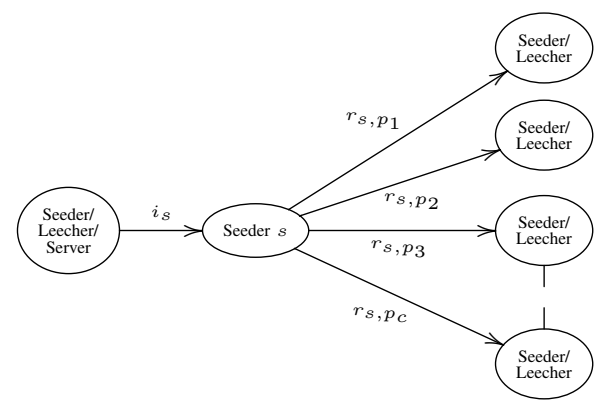

Fig. 1. Basic principle of live seeding

The efficiency of a set $X \subseteq S$ is defined in the same way: we consider the difference between what comes out of $X$ and what enters, all reported to capacity:

$\eta_{d}(X):=\frac{\sum_{s \in X, q \in\{L, S \backslash X\}} r_{s, q}-\sum_{p \in\{C, L, S \backslash X\}, s \in X} r_{p, s}}{U_{X}}$.

By considering (1), (6), (7) and $\sum_{s, t \in X} r_{s, t}$, we obtain a more compact expression for $\eta_{d}$ :

$$
\eta_{d}(X)=\frac{\sum_{s \in X} \eta_{d}(s) u_{s}}{U_{X}}
$$

Equation (8) tells that $\eta_{d}(X)$ can be seen as the weighted average of the seeders individual efficiencies.

\footnotetext{
${ }^{2}$ Deciding which peers involved in $i_{s}$ are responsible for the "waste" is arbitrary, and one could decide to subtract $i_{s}$ from the bandwidth of the sending peers. However, making the receiving seeder responsible for its own input rate makes the analysis simpler.
} 


\section{A. Optimal efficiency}

The optimal efficiency $\eta_{O P T}(s)$ of a seeder $s$ in a given system is defined as the supremum of the efficiencies it can get over all possible diffusion schemes.

$$
\eta_{O P T}(s)=\sup _{d}\left(\eta_{d}(s)\right)
$$

$\eta_{O P T}(s)$ is an upper bound for the proportion of the upload bandwidth that can be useful for that system.

The same definition stands for the optimal efficiency of any subset $X \subseteq S$ :

$$
\eta_{O P T}(X)=\sup _{d}\left(\eta_{d}(X)\right)
$$

There is no guarantee that the individual optimal efficiencies of seeders can be aggregated, because they may be reached for distinct schemes (a counter-example is given in Section V). As a consequence, Equation (8) becomes an inequality when considering optimal efficiency:

$$
\eta_{O P T}(X) \leq \frac{\sum_{s \in X} \eta_{O P T}(s) u_{s}}{U_{X}} .
$$

For convenience, subscripts may be omitted when there is no ambiguity. We may also use metonymic notation in order not to clutter notation: $\eta(y)$ may denote the efficiency of a seeder characterised by some property $y$ (like the input rate, upload bandwidth, fanout, ...).

\section{Perfect And Limited Fanout Systems}

In this section, we derive the optimal efficiency of seeders when there is no explicit overhead.

\section{A. Perfect systems}

The optimal performance of seeders in a perfect system is given by the following theorem:

Theorem 1. The optimal efficiency of a subset $X \subseteq S$ of seeders is

$$
\eta(X)=\left(1-\frac{1}{N_{L}}\right) \min \left(1, \frac{N_{L} r}{U_{X}}\right) .
$$

Proof: First we give a scheme that achieves the efficiency given by (12). The scheme is the following: each seeder $s \in X$ receives from the servers a distinct substream of rate $\frac{u_{s}}{N_{L}}$ (if $U_{X} \leq N_{L} r$ ) or $\frac{u_{s}}{U_{S}} r$ (otherwise), and broadcasts that substream to the $N_{L}$ leechers. Under that scheme, the input received by $X$ from nodes outside $X$ is $\min \left(\frac{U_{X}}{N_{L}}, r\right)$, and the output given to leechers is $\min \left(U_{X}, N_{L} r\right)$. Subtracting the input from the output and dividing by $U_{X}$ gives the efficiency $\eta(X)$ from (12).

Then, we need to prove that $\eta(X)$ cannot be greater than $\left(1-\frac{1}{N_{L}}\right) \min \left(1, \frac{N_{L} r}{U_{X}}\right)$. If $I_{X}$ is the input received by $X$ in a given scheme, the corresponding useful output cannot be more that $\min \left(U_{X}, \min \left(I_{X}, r\right) N_{L}\right)$ because :

- $U_{X}$ is the capacity of $X$;

- $\min (I, r)$ is the maximal rate of information that $X$ can get. The best it can achieve is to send that rate to the $N_{L}$ leechers: sending it to more peers, for instance seeders outside $X$, would be ineffective because all leechers already get the information received by $X$.

Given the input and output rates, and according to Equation (7), the efficiency of $X$ for a given input $I_{X}$ is bounded by

$$
\min \left(1, I_{X} \frac{N_{L}}{U_{X}}, r \frac{N_{L}}{U_{X}}\right)-\frac{I_{X}}{U_{X}} .
$$

We deduce that the optimal efficiency is bounded by

$$
\sup _{I_{X} \geq 0}\left(\min \left(1, I_{X} \frac{N_{L}}{U_{X}}, r \frac{N_{L}}{U_{X}}\right)-\frac{I_{X}}{U_{X}}\right) .
$$

If $U_{X} \leq N_{L} r$, we get a maximal efficiency $1-\frac{1}{N_{L}}$ for $I_{X}=$ $\frac{U_{X}}{N_{L}}$, and if $U_{X} \geq N_{L} r$, we get $\frac{r\left(N_{L}-1\right)}{U_{X}}$ for $I_{X}=r$. Therefore the efficiency is never more than $\left(1-\frac{1}{N_{L}}\right) \min \left(1, \frac{N_{L} r}{U_{X}}\right)$. This concludes the proof.

Note that the condition $U_{X}>N_{L} r$ corresponds to an overprovisioned system, where seeders from $X$ have more bandwidth than required to feed the stream $r$ to all leechers by themselves. In the definition we proposed, efficiency is normalized by the dedicated upload bandwidth, so overprovisioned systems naturally have lower efficiencies.

If we restrict to non-overprovisioned subsets $X$, Equation (12) simplifies to

$$
\eta(X)=1-\frac{1}{N_{L}} .
$$

In other words, seeders are asymptotically optimal in a perfect P2P live streaming system. The explanation is that the only bandwidth waste boils down to at most one streamrate redirected to them for replication.

\section{B. Limited fanout}

Each seeder $s$ has now a limited fanout $c_{s}$. Without loss of generality, we assume that $\forall s \in S, c_{s} \leq N_{L}$.

Theorem 2. The optimal efficiency of a single seeder $s \in S$ with limited connections $c_{s}$ is

$$
\eta(s)=\left(1-\frac{1}{c_{s}}\right) \min \left(1, \frac{r c_{s}}{u_{s}}\right) .
$$

In particular, if $r c_{s} \geq u_{s}$ (the fanout is high enough for allowing to use all the upload of s), we just have

$$
\eta(s)=1-\frac{1}{c_{s}} \text {. }
$$

Proof: As $s$ cannot reach more than $c_{s}$ peers, we just consider a sub-system made of $C, s$ and $c_{s}$ leechers, and we conclude by applying Theorem 1, with $c_{s}$ instead of $N_{L}$.

Unlike perfect systems, this result only applies for one single seeder, with no simple extension for a set of seeders. Equation (11) can be a strict inequality, meaning that efficiency is lost in the process of making multiple seeders work together. Consider for instance a toy system made of $N_{L}=3$ leechers and two seeders $s_{1}$ and $s_{2}$ with parameters $u_{1}=\frac{3}{2} r, c_{1}=2$, $u_{2}=r, c_{2}=3$. Using Equation (14), we get $\eta_{O P T}\left(s_{1}\right)=\frac{1}{2}$ and $\eta_{O P T}\left(s_{2}\right)=\frac{2}{3}$, so

$$
\frac{\eta_{O P T}\left(s_{1}\right) u_{1}+\eta_{O P T}\left(s_{2}\right) u_{2}}{u_{1}+u_{2}}=\frac{17}{30} .
$$


But if we try to find a scheme that maximize the efficiency of $\left\{s_{1}, s_{2}\right\}$, the best solution leads to

$$
\eta_{O P T}\left(\left\{s_{1}, s_{2}\right\}\right)=\frac{8}{15}<\frac{17}{30} .
$$

The good news is that for specific scenarios, we can guarantee that $\eta_{O P T}(X)=\frac{\sum_{s \in X} \eta_{O P T}(s) u_{s}}{U_{X}}$. This is for instance the case when $X$ is proportionally homogeneous.

Theorem 3. Consider a set $X \subseteq S$ that is proportionally homogeneous, i.e. there is a rate e so that $u_{s}=e c_{s}$ for all $s \in X$. Then, for $N_{X} \leq\left\lfloor\frac{N_{L}-1}{\max _{s} \in X\left(c_{s}\right)-1}\right\rfloor\left\lfloor\frac{r}{e}\right\rfloor$

$$
\eta_{O P T}(X)=\frac{\sum_{s \in X}\left(1-\frac{1}{c_{s}}\right) u_{s}}{U_{X}}=\frac{\sum_{s \in X} \eta_{O P T}(s) u_{s}}{U_{X}} .
$$

Note that $e \leq r$ is an implicit condition of Theorem 3: otherwise, the result only apply for $N_{X} \leq 0$ (empty set).

Corollary 1. If all seeders in $X$ have the same upload $u$, maximal fanout $c$, and if $N_{X} \leq\left\lfloor\frac{N_{L}-1}{c-1}\right\rfloor\left\lfloor\frac{c r}{u}\right\rfloor$, then

$$
\eta_{O P T}(X)=1-\frac{1}{c} \text {. }
$$

Remark: In the homogeneous case, if we neglect truncation effects, the condition of Corollary 1 corresponds to $U_{X} \leq\left(N_{L}-1\right) r \frac{c}{c-1}$. As $\left(N_{L}-1\right) \frac{c}{c-1} \geq N_{L}$ (because $c \leq N_{L}$ ), we get the sufficient condition $U_{X} \leq r N_{L}$. Therefore, Corollary 1 can be interpreted as follows: in the homogeneous limited fanout model, up to truncation effects, efficiencies can be aggregated without loss for any nonoverprovisionned subset $X$.

Proof: Given Equations (11) and (15), we just need to give a diffusion scheme $d$ such that $\eta_{d}(X)=\frac{\sum_{s \in X} \eta_{O P T}(s) u_{s}}{U_{X}}$.

That diffusion scheme is the following: the streamrate $r$ is divided into $\left\lfloor\frac{r}{e}\right\rfloor$ distinct substreams of rate $e$. We then build up to $\left\lfloor\frac{r}{e}\right\rfloor$ trees such that: each seeder $s$ in $X$ is an internal node for exactly one tree, having exactly $c_{s}=\frac{u_{s}}{e}$ children; the leaves are taken among the leechers; a leecher can belong to several trees, but is contained at most once per tree.

A given tree can have up to $N_{L}$ leaves, but no more. We deduce that one tree can contain $\left\lfloor\frac{N_{L}-1}{\max _{s \in X}\left(c_{s}\right)-1}\right\rfloor$ seeders, because a tree with $k$ internal nodes (from $X$ ) has at most $k\left(\max _{s \in X}\left(c_{s}\right)-1\right)+1$ leaves. So the rules of the scheme can be respected if $N_{X} \leq\left\lfloor\frac{N_{L}-1}{c-1}\right\rfloor\left\lfloor\frac{c r}{u}\right\rfloor$.

In the corresponding diffusion scheme, where each tree is used to transmit one of the $\left\lfloor\frac{r}{e}\right\rfloor$ distinct substreams of rate $e$, we verify that each seeder $s$ works at optimal efficiency $1-\frac{1}{c_{s}}$. Equation (8) concludes the theorem. The corollary is just a special case where $e=\frac{u}{c}$ and $\max _{s \in X}\left(c_{s}\right)=c$.

Remark: The proof shows that the bound on $N_{X}$ is actually related to the numbers of seeders that can fit in a tree with the constraints that each seeder $s$ is an internal node with exactly $c_{s}$ children and there are no more than $N_{L}$ leaves. The bound we gave is very conservative, because it assumes $\max _{s \in X}\left(c_{s}\right)$ children for all seeders. It may not be tight, especially if $c_{s}$ spans a wide range. However, finding out the tight maximal number of seeders under heterogeneous fanout is difficult, as it is equivalent to solving a multiple knapsack problem.

\section{EXPLICIT OVERHEAD}

From now on, we will focus on the explicit linear overhead model, with proportional cost $a$ and additive cost $b$. Under this model, the bandwidth required for sending one copy of the stream through a single connection is $R:=(1+a) r+b$. One easily checks that $\eta_{\max }:=\frac{r}{R}$ is the maximal efficiency achievable for any peer (leecher or seeder).

Unless otherwise stated, numerical examples will take as default parameters $r=100 \mathrm{KBytes} / \mathrm{s}$, a proportional overhead of $10 \%(a=0.1)$, and two possible additive costs, small $(b=$ $1.7 \mathrm{KBytes} / \mathrm{s})$ and large $(b=25 \mathrm{KBytes} / \mathrm{s})$. Most figures will display the relative efficiency $\eta / \eta_{\max }$ instead of $\eta$, in order to facilitate the comparison between the two overhead settings.

\section{A. Efficiency of a single seeder: main theorem}

The following theorem gives the optimal efficiency of one single seeder when the overhead is linear.

Theorem 4. Assuming a linear overhead supported by the sender, optimal efficiency of a seeder $s$ is $\eta(s)=\frac{\left(N_{L}-1\right) r}{u_{s}}$ for $u_{s} \geq N_{L} R$. For $u_{s}<N_{L} R$, we have

$$
\begin{aligned}
\eta(s)= & \begin{cases}0 & \text { if } 0 \leq u_{s} \leq 2 b, \\
\frac{\left(1-\sqrt{\frac{b}{u_{s}}}\right)^{2}}{1+a}-\epsilon_{1}\left(u_{s}\right) & \text { if } 2 b \leq u_{s} \leq \frac{R^{2}}{b}, \\
\frac{r}{R}-\frac{r}{u_{s}}-\epsilon_{2}\left(u_{s}\right) & \text { if } u_{s} \geq \frac{R^{2}}{b}, \text { with }\end{cases} \\
& \left\{\begin{array}{l}
0 \leq \epsilon_{1}\left(u_{s}\right) \leq \frac{1}{1+a}\left(\frac{b}{u_{s}}\right)^{\frac{3}{2}}, \\
0 \leq \epsilon_{2}\left(u_{s}\right) \leq \frac{1}{1+a} \frac{b}{u_{s}} \leq \frac{1}{1+a}\left(\frac{b}{R}\right)^{2} .
\end{array}\right.
\end{aligned}
$$

Proof: The part $u_{s} \geq N_{L} R$ is straightforward. It corresponds to an overprovisioned situation where $s$ alone can provide the live content to all leechers. This is the optimal scheme for $s$, leading to $\eta(s)=\frac{\left(N_{L}-1\right) r}{u_{s}}$.

Equation (18), which corresponds to the case $u_{s}<N_{L} R$, can be proved in three steps:

- Finding the maximal efficiency for a given bandwidth $u$ and fanout $c$;

- Maximizing the corresponding equations for a continuous $c$;

- Bounding the gap induced by the fact that $c$ has to be an integer.

1) Maximizing $\eta$ for given $u, c$ : we first notice that for achieving maximal efficiency, all output rates have to be equal to the input rate: if it is not the case in a given scheme, replacing all output rates by their average value allows to reduce the input rate to that average value (it had to be greater than the maximal output in the original case), increasing efficiency. Therefore the optimal efficiency must be of the form $\eta(s)=\frac{(c-1) e}{u}$, for some rate $0 \leq e \leq r$. It is then obvious that one have interest to choose the highest value of $e$ that is feasible. 
Note that if $c=1$, the seeder can only replicate its input and has null efficiency; the seeder needs to maintain at least 2 connections with spared bandwidth to have a nonnull efficiency. This settles that $\eta=0$ for $u \leq 2 b$. Otherwise, two cases are to be considered:

- if $c$ is the bottleneck (this happens for $u \geq R c$ ), then $s$ has enough bandwidth to broadcast the whole stream $r$ to $c$ targets, achieving efficiency $\frac{(c-1) r}{u}$;

- if $u$ is the bottleneck (for $u<R c$ ), then the optimal input rate $e$ is solution of $c((1+a) e+b)=u$, leading to $e=\frac{\frac{u}{c}-b}{1+a}$. Corresponding efficiency is

$$
\begin{aligned}
\eta & =\frac{(c-1) e}{u}=\frac{(c-1)\left(\frac{u}{c}-b\right)}{(1+a) u} \\
& =\frac{1-\frac{1}{c}-\frac{b}{u}(c-1)}{1+a}
\end{aligned}
$$

For $u<R N_{L}$, the bottleneck is necessary one of the above, so we deduce that the optimal efficiency for given $u$ and $c$ is

$$
\eta(u, c)=\min \left(\frac{(c-1) r}{u}, \frac{\left(1-\frac{1}{c}\right)-\frac{b}{u}(c-1)}{1+a}\right)
$$

2) Maximizing $\eta$ for given $u$ : We now see (19) as a function of $c$ and try to find its maximal value. We propose to first solve the problem in $\mathbb{R}$ before considering integers.

We introduce

$$
\begin{aligned}
\eta_{1}(c) & :=\frac{(c-1) r}{u} \text { and } \\
\eta_{2}(c) & :=\frac{\left(1-\frac{1}{c}\right)-\frac{b}{u}(c-1)}{1+a} .
\end{aligned}
$$

The two functions have the following properties:

- $\eta_{1}$ is always increasing, and positive for $c \geq 1$;

- $\eta_{2}$ goes to $-\infty$ for $c$ going to 0 and $+\infty$. It has a unique maximum $\frac{\left(1-\sqrt{\frac{b}{u_{s}}}\right)^{2}}{1+a}$, which is reached for $c=\sqrt{\frac{u}{b}}$

- $\eta_{1}=\eta_{2}$ for $c=1$ (corresponding efficiency is 0 ) and $c=\frac{u}{R}$ (corresponding efficiency is $\frac{r}{R}-\frac{r}{u}$ ).

We deduce that the optimal efficiency for given $c, \eta=$ $\min \left(\eta_{1}, \eta_{2}\right)$ is equal to $\eta_{1}$ for $1 \leq c \leq \frac{u}{R}$ and $\eta_{2}$ for $c \geq \frac{u}{R}$. Two cases are then to be considered:

- if $\sqrt{\frac{u}{b}} \leq \frac{u}{R}$ (that is $u \geq \frac{R^{2}}{b}$ ), then $\eta$ is increasing for $1 \leq$ $c \leq \frac{u}{R}$, decreasing for $c \geq \frac{u}{R}$. The maximal efficiency is therefore $\frac{r}{R}-\frac{r}{u}$, reached for $c=\frac{u}{R}$;

- if $\sqrt{\frac{u}{b}} \geq \frac{u}{R}$ (that is $u \leq \frac{R^{2}}{b}$ ), then the maximal efficiency is the one of $\eta_{2}, \frac{\left(1-\sqrt{\frac{b}{u_{s}}}\right)^{2}}{1+a}$, reached for $c=\sqrt{\frac{u}{b}}$.

3) Bounding the quantification gap: While the optimal value $c_{O P T}$ we found is a real number, only integer value are eligible. However, as the function $\eta=\min \left(\eta_{1}, \eta_{2}\right)$ always admits a unique maximum, the effective optimal efficiency $\eta(s)$ is necessarily $\max \left(\eta\left(\left\lfloor c_{O P T}\right\rfloor\right), \eta\left(\left\lceil c_{O P T}\right\rceil\right)\right)$. In particular, we have $\eta\left(c_{O P T}+1\right) \leq \eta(s) \leq \eta\left(c_{O P T}\right)$, from which we deduce

$\eta(s)=\eta\left(c_{O P T}\right)-\epsilon$, with $0 \leq \epsilon \leq \eta\left(c_{O P T}\right)-\eta\left(c_{O P T}+1\right)$

From there, noticing that $\eta=\eta_{2}$ for $c \geq c_{O P T}$, we get

$$
\eta\left(c_{O P T}\right)-\eta\left(c_{O P T}+1\right)=\frac{\frac{b}{u}-\frac{1}{c_{O P T}\left(c_{O P T}+1\right)}}{1+a} .
$$

- If $u \leq \frac{R^{2}}{b}$, then $c_{O P T}=\sqrt{\frac{u}{b}}$, so we get

$$
\begin{aligned}
\eta\left(c_{O P T}\right)-\eta\left(c_{O P T}+1\right) & =\frac{\frac{b}{u}\left(1-\frac{1}{1+\sqrt{\frac{b}{u}}}\right)}{(1+a)} \\
& \leq \frac{\left(\frac{b}{u}\right)^{\frac{3}{2}}}{(1+a)} ;
\end{aligned}
$$

- if $u \geq \frac{R^{2}}{b}$, we just use

$$
\eta\left(c_{O P T}\right)-\eta\left(c_{O P T}+1\right) \leq \frac{b}{u(1+a)},
$$

and note that $\frac{b}{u} \leq\left(\frac{b}{R}\right)^{2}$. This concludes the proof.

\section{B. Efficiency of a single seeder: discussion}

Following theorem 4 and proof, the following remarks can be made.

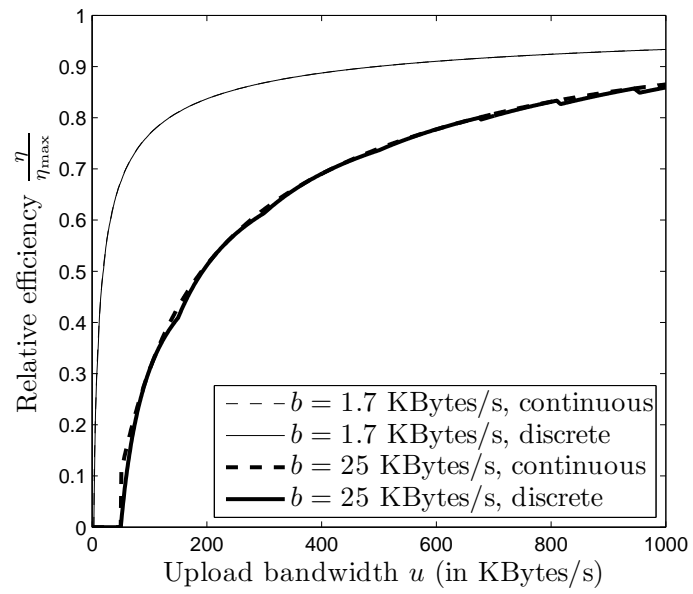

Fig. 2. Validity of the continuous approximation of the optimal efficiency

1) Closed formulas approximation: the $\epsilon_{1}$ and $\epsilon_{2}$ terms are negligible as long as $u_{s}$ is big enough compared to the additive cost $b$, so in most cases, one can safely use the continuous optimum $\eta\left(c_{O P T}\right)$ (step 2) of the proof) instead of the discrete one $\max \left(\eta\left(\left\lfloor c_{O P T}\right\rfloor\right), \eta\left(\left\lceil c_{O P T}\right\rceil\right)\right)$. In other words,

$$
\eta(s) \approx \begin{cases}\frac{\left(1-\sqrt{\frac{b}{u_{s}}}\right)^{2}}{1+a} & \text { if } 2 b \leq u_{s} \leq \frac{R^{2}}{b}, \\ \eta_{\max }-\frac{r}{u_{s}} & \text { if } u_{s} \geq \frac{R^{2}}{b} .\end{cases}
$$

To illustrate the validity of this approximation, Figure 2 compares it to the exact efficiency for the two numerical settings we proposed at the beginning of this Section. We can see that the difference is barely noticeable for a large additive overhead, and invisible for a small one. 
2) Low/medium bandwidth: The case $u_{s} \leq \frac{R^{2}}{b}$ can be interpreted as the upload bandwidth is no more than $\frac{R}{b}$ times the rate $R$. In most practical situations, one would expect $b \ll R$, so most seeders would probably fall in this case, which corresponds to low, medium and reasonably high bandwidths.

Within this range, it is interesting to note that both the optimal number of connection and corresponding efficiency are independent of $r$. Moreover, one can note that the number of connections, $\sqrt{\frac{u_{s}}{b}}$, is quite similar to the empirical formula used in the current BitTorrent mainline client, $\sqrt{0.6 u}$ [6]. This makes us think that the results given here could be adapted to other scenarios than live seeding (this would need to be further investigated in a future work). The 0.6 factor would corresponds to an additive connection cost $b \approx 1.7 \mathrm{KBytes} / \mathrm{s}$, which explains why we use this value as one of our numerical settings (the other value, $b=25 \mathrm{KBytes} / \mathrm{s}$, is totally arbitrary).

3) (Very) high bandwidth: For very high bandwidths (corresponding for instance to nodes managed by a content distribution network), the efficiency tends to $\eta_{\max }$ as $u_{s}$ goes to infinity under the assumption that the scenario is not overprovisioned (i.e. $\frac{u_{s}}{N_{L}}<R$ ): super-seeders can asymptotically reach the best achievable efficiency given the overhead constraints.

4) Importance of input shaping: Seeders do not need to get the whole streamrate. This fact allows to adjust their input rate as desired, which is a key to achieve optimal efficiency.

For instance, under the assumption that the input rate of a seeder $s$ is $r$, one easily checks that its best achievable efficiency is

$$
\eta_{r}(s)=\max \left(0, \frac{r\left(\left\lfloor\frac{u_{s}}{R}\right\rfloor-1\right)}{u_{s}}, \frac{1-\frac{b}{u_{s}}\left\lceil\frac{u_{s}}{R}\right\rceil}{1+a}-\frac{r}{u_{s}}\right)
$$

(the case 0 corresponds to situations where the best choice is not to use $s$, saving the input rate).

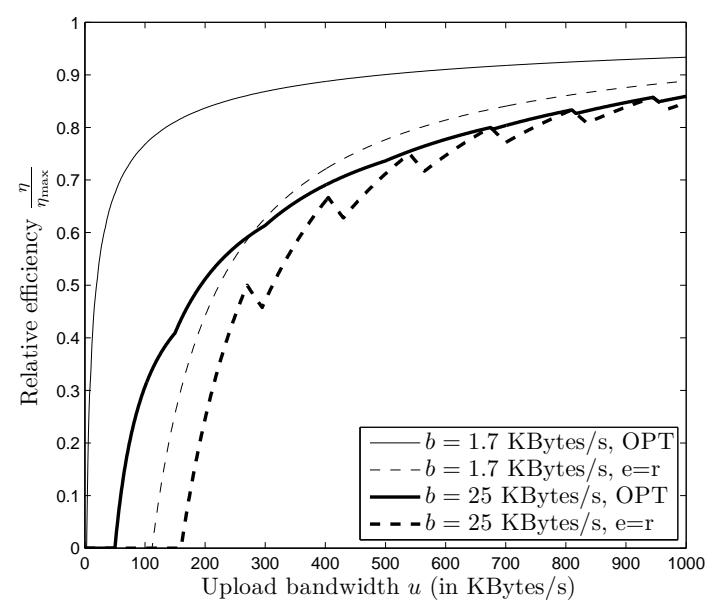

Fig. 3. Impact of a badly shaped input rate

Figure 3 gives a graphical comparison of $\eta_{O P T}$ and $\eta_{r}$. While seeders with optimized input rates can get a decent efficiency starting from a few $b$ 's of upload bandwidth, if the input is $r$, seeders with an upload bandwidth less than

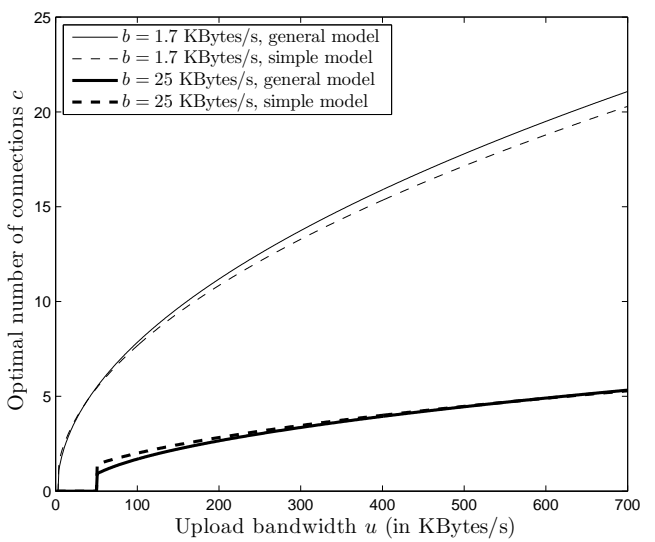

Fig. 4. General overhead model vs simple overhead model

$R$ are totally inefficient (they cannot give more than they receive, so the best choice is not to use them). We also notice that the difference remains important even for higher upload bandwidth, especially if the additive overhead is small.

5) About receiver-side overhead: In our model, we made the assumption that the burden of the overhead was only on the sender. A more general model would consist in assuming that in addition to the sender overhead of parameters $(a, b)$, there is a receiver overhead of parameters $\left(a_{r}, b_{r}\right)$ (if $p$ receives a streamrate $r_{q, p}$ from $q$, it has to use an upload bandwidth $\left.a_{r} r_{q, p}+b_{r}\right)$.

Theorem 4 and proof can be adapted to the general model, at the price of increased complexity. For instance, in the medium range scenario $\left(2 b<u_{s} \leq \frac{R^{2}}{b}\right)$, we have an optimal (continuous) number of connections

$$
c_{O P T}=\sqrt{\frac{u_{s}}{b}} .
$$

In the general model, this would become

$c_{O P T}=\frac{-a_{r} b+\sqrt{b\left(a+a_{r}+1\right)\left(u-b_{d}-a b_{r}+a_{r} b+a u\right)}}{b(a+1)}$.

We see that formulas get much more complex in the general model. However, if one compares the practical values given by (22) and (23), we see that the general behavior remains practically the same. This is depicted in Figure 4 (receiver overhead is assumed to be the same that the sender overhead, i.e. $a_{r}:=a$ and $b_{r}:=b$ ).

As the added complexity does not seem to bring lot of practical difference, we choose to discard the receiver overhead in our model. However, the reason we can do that is probably that the natural use of live seeders is to feed them with a single input rate, which reduce the impact of receiver overhead. If we want to extend our framework to leechers, which usually receive multiple substreams from multiple sources, a proper modeling of the receiver overhead may become mandatory. 


\section{Efficiency of a set of seeders}

Like for the limited fanout model, there is no guarantee that the optimal single efficiencies of seeder can be aggregated in a common scheme. In the following, we propose two heuristics that allow to somehow adapt Theorem 3 to the overhead model: the mono-rate and dichotomic rates diffusion schemes.

1) Mono-rate scheme: The idea of the mono-rate approach is somehow simple: if a set of seeders agree to a common substream rate $e$, they can behave as a proportionally heterogeneous set. Their efficiency obeys to the following theorem:

Theorem 5. Consider a set $X \subseteq S$ that verifies:

- $\bar{u}_{X} \leq \frac{2 R^{2}}{b}$;

- $N_{X} \leq\left\lfloor\frac{N_{L}-1}{\left\lfloor\frac{\max _{s \in X}\left(u_{s}\right)}{E}\right\rfloor-1}\right\rfloor\left\lfloor\frac{R-b}{E-b}\right\rfloor$, with $E=\sqrt{\frac{b \bar{u}_{X}}{2}}$.

Then, if all seeders on $X$ agree on a common rate $e:=\frac{E-b}{1+a}$ used for all inputs and outputs, the efficiency $\eta_{e}(X)$ of the corresponding scheme verifies

$$
\frac{\left(1-\sqrt{\frac{2 b}{\bar{u}_{X}}}\right)^{2}}{1+a}<\eta_{e}(X) \leq \frac{\left(1-\sqrt{\frac{b}{\bar{u}_{X}}}\right)^{2}}{1+a}
$$

Proof: Consider a given rate $e \leq r$. Call $E:=(1+a) e+b$ the corresponding rate with overhead. The maximal efficiency of a seeder $s$ having $e$ as input and ouputs is reached when $s$ opens the maximal number of outgoing connections allowing to stream $e$. This leads to

$$
\eta_{e}(s)=\frac{\left(\left\lfloor\frac{u_{s}}{E}\right\rfloor-1\right) e}{u_{s}} .
$$

In particular,

$$
\frac{e}{E}-2 \frac{e}{u_{s}}<\eta_{e}(s) \leq \frac{e}{E}-\frac{e}{u_{s}} .
$$

Assume that the number of seeders in $X$ is small enough to allow perfect aggregation of efficiencies, like for Theorem 3 (the corresponding condition will be derived later). We then have $\eta_{e}(X)=\frac{\sum_{s \in X} \eta_{e}(s) u_{s}}{U_{X}}$, therefore

$$
\frac{e}{E}-2 \frac{e}{\bar{u}_{X}}<\eta_{e}(X) \leq \frac{e}{E}-\frac{e}{\bar{u}_{X}} .
$$

The maximal value of $\frac{e}{E}-\frac{e}{\bar{u}_{X}}$ is $\frac{\left(1-\sqrt{\frac{b}{\bar{u}_{X}}}\right)^{2}}{1+a}$, proving the right part of (24). The maximal value of $\frac{e}{E}-2 \frac{e}{\bar{u}_{X}}$ is $\frac{\left(1-\sqrt{\frac{2 b}{\bar{u}_{X}}}\right)^{2}}{1+a}$, and it is reached for $E=\sqrt{\frac{b \bar{u}_{X}}{2}}$. As we have $E \leq R$, this implies $\bar{u}_{X} \leq \frac{2 R^{2}}{b}$.

We then need to give a sufficient condition for aggregating the efficiencies without losses. We can use the condition from Theorem 3, $N_{X} \leq\left\lfloor\frac{N_{L}-1}{\max _{s \in X}\left(c_{s}\right)-1}\right\rfloor\left\lfloor\frac{r}{e}\right\rfloor$. Noticing that $c_{s}=$ $\left\lfloor\frac{u_{s}}{E}\right\rfloor$ allows to conclude.

2) Dichotomic scheme: The dichotomic approach consists in the diffusion of several substreams whose rates are dividers of $r$, instead of using a single rate $e$. In details, the predetermined substreams are:

- The video stream of rate $r$, which can be split into

- 2 non-overlapping substreams of rate $\frac{r}{2}$, each of which can be split into 2 substreams

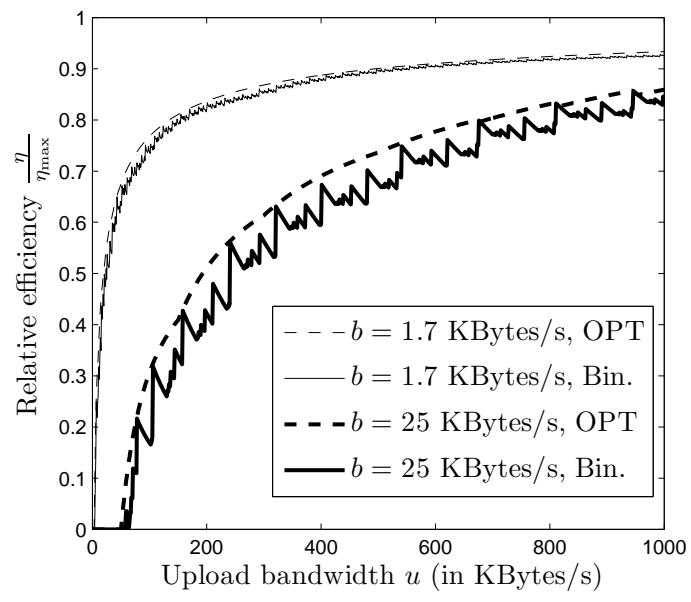

Fig. 5. Dichotomic vs optimal individual efficiencies

- ...

- $2^{k_{\max }}$ non-overlapping substreams of rate $\frac{r}{2^{k_{\max }}}$, for some $k_{\max } \geq 0$.

$k$ is called the level of a substream of rate $\frac{r}{2^{k}}$

A seeder $s$ is said to operate at level $k$ if it behaves as follows:

- it receives as input a level $k$ substream; let $l:=k$ be his working level;

- As long as $s$ has a residual upload bandwidth greater than $b$ and $l \leq k_{\max }$, do:

- if there is not enough residual upload bandwidth to establish a new output of level $l$,

- then $l=l+1$ (a children substream of the current level $l$ substream is chosen),

- else create a new output of level $l$.

The corresponding efficiency is denoted $\eta_{k}(s)$. In order to optimize the dichotomic approach, each seeder operates at a level that maximizes its single efficiency, i.e. chooses a level $k_{s}$ such that $\eta_{k_{s}}(s)=\max _{0 \leq k \leq k_{\max }} \eta_{k}(s)$. The corresponding efficiency is denoted $\eta_{B i n}(s)$.

As the operating rate is necessarily a divider of $r, \eta_{B i n}(s)$ is necessarily suboptimal. However, the different levels allow enough freedom to get an efficiency close enough to be optimal. For instance, Figure 5 gives a graphical comparison of $\eta_{B i n}(s)$ and $\eta_{O P T}(s)$, using $k_{\max }=\left\lfloor\log _{2}\left(\frac{r}{b}\right)\right\rfloor$ (this is an arbitrary choice that corresponds to stopping the subdivision when substreams need more overhead that their actual goodput). One observes that the individual efficiency loss is quite sustainable, especially for a low additive overhead.

For a given set $X$ of seeders, the construction of a dichotomic diffusion scheme is rather simple:

- all seeders operating at level $k$ organize to achieve up to $2^{k}$ diffusions tree for the level $k$; each seeder try to join the level $k$ diffusion tree which currently possesses less leaves.

- if a level $k$ seeder has outputs of level $k^{\prime}>k$, they can either be directly transmitted to leechers or serve as root 
for a level $k^{\prime}$ diffusion tree;

- if some seeders at level $k$ miss the input streamrate to build their diffusion scheme, they may use a leaf from a parent substream diffusion tree (some of parent rate will be wasted).

Under some conditions, we can evaluate the efficiency of $X$ under a dichotomic diffusion.

Theorem 6. If, for a given set $X \subseteq S$, we have $U_{X} \leq N_{L} R$, and if all non-empty diffusion trees can be rooted with proper input, then the efficiency $\eta_{B i n}(X)$ of $X$ under a dichotomic diffusion verifies

$$
\frac{\sum_{s \in X} \eta_{B i n}(s) u_{s}}{U_{X}}-\frac{r k_{\max }}{U_{X}} \leq \eta_{B i n}(X) \leq \frac{\sum_{s \in X} \eta_{B i n}(s) u_{s}}{U_{X}}
$$

The interpretation is the following: up to a term $\frac{r k_{\max }}{U_{X}}$, which is small if $U_{X}$ is big enough, the individual dichotomic efficiencies, which are close to the optimal individual efficiencies, can be aggregated without loss.

Proof: The condition $U_{X} \leq N_{L} R$ ensures that no diffusion tree has more leaves than there are leechers in need of the corresponding substream. This can be shown by induction:

- at level 0 , the diffusion tree cannot have more than $\left\lfloor\frac{U_{X}}{R}\right\rfloor$ leaves, which is smaller than $N_{L}$.

- at level $k$, let $U_{k}$ denote the bandwidth that remains after the bandwidth consumed from lower level is substracted; let $N_{k}$ the maximal number of leechers that can be leaves at that level (a given leecher is counted with multiplicity equal to the number the level $k$ substream it needs; let $M_{k}$ the number of leechers that get a level $k$ substream (with multiplicity). Note the relation $N_{k}=2\left(N_{k-1}-M_{k-1}\right)$, i.e. the maximal number at a given level is twice the slots that have not been filled in the previous level. Assume that $U_{k-1} \leq N_{k-1}\left((1+a) \frac{r}{2^{k-1}}+b\right)$, that is at level $k-1$, the residual bandwidth is not overprovisioned compared to the number of possible leaves Then we have

$$
\begin{aligned}
U_{k} & \leq U_{k-1}-M_{k-1}\left((1+a) \frac{r}{2^{k-1}}+b\right) \\
& \leq\left(N_{k-1}-M_{k-1}\right)\left((1+a) \frac{r}{2^{k-1}}+b\right) \\
& \leq N_{k}\left((1+a) \frac{r}{2^{k}}+\frac{b}{2}\right) \leq N_{k}\left((1+a) \frac{r}{2^{k}}+b\right)
\end{aligned}
$$

So at any given level, a diffusion tree can always find a leecher to give its output to. Therefore the only waste compared with individual efficiencies lies when the root input of a tree comes from a parent substream. This is bounded by $r$ when considering all roots at a given level $k>0$, leading to a total waste bounded by $r k_{\max }$. Normalizing by $U_{X}$ concludes the proof.

3) Comparison of the two methods: The mono-rate approach is simple to describe, which makes it a good proof of concept of using multiple seeders in a system with overhead. However, the dichotomic approach, although more complex, has many advantages over the mono-rate approach that make it more suitable for a practical use.
Firstly, the substreams are pre-determined, while mono-rate requires to determine the proper input rate $e$, which depends on $\bar{u}_{X}$. Among other things, this facilitate considerably the interaction with the leechers' diffusion process. Furthermore, under the dichotomic approach, a seeder $s$ can determine its operating level by itself (it is just a function of $u_{s}$ ) while in the mono-rate approach, knowing $\bar{u}_{X}$ implies some knowledge of the whole set $X$. This is even worse when considering dynamics in $X$ : A change in $e=f\left(\bar{u}_{X}\right)$ requires a complete upset of the diffusion trees in the mono-rate approach, while changes are expected to be mostly local in the dichotomic approach.

Also note that as streamrate are dividers of $r$, the quantification effect $\left\lfloor\frac{r}{e}\right\rfloor$ that may limit the mono-rate approach (cf Theorem 5) has no equivalent in the dichotomic approach.

Finally, the mono-rate approach can force lot of seeders to use an input rate that is far from the single seeder optimal. This impact is bounded ( $\mathrm{cf}$ Theorem 5), but can be non negligible, especially if the seeders' bandwidths are highly heterogeneous. In contrast, the dichotomic approach adjusts afor each seeder $s$ a level $k_{s}$ such that the input rate is to far from the optimal.

\section{DISCUSSION}

\section{A. Leecher diffusion process}

We did not consider in details the way to make the diffusion processes of leechers and seeders work together. This is a problem in itself, which deserves a separate study. The study performed in [3] seems to be adaptable to the case with seeders, at least for the limited fanout model, but a further work is required to transpose the results to the overhead model (including keeping in mind the existence of receiverside overhead).

However, we argue that knowing how to optimize the diffusion process of seeders alone is not a bad starting point.

\section{B. Make a minimal use of seeders}

While all this paper is devoted to make the best possible use of seeders, we should recall that in the design of a real system, targeting the maximal seeder efficiency is not necessarily the smartest thing to do.

In fact, seeders "waste" their input rate by design, which makes them inherently less efficient that leechers. Therefore, the secret of wise seeding is to use it when leechers are not enough, and not in replacement of leechers:

- Try to achieve the most of the content diffusion by using the servers and leechers alone. If possible, the leechers should perform a lossless diffusion of a common substream of rate $r^{\prime} \leq r$ among all of them instead of a partial or lossy diffusion of rate $r$;

- if $r^{\prime}<r$, use seeders to finish the job, achieving a rate what would not have been possible otherwise. This is were the results of this paper apply, which describe the best one can expect from seeders and how to get it. 


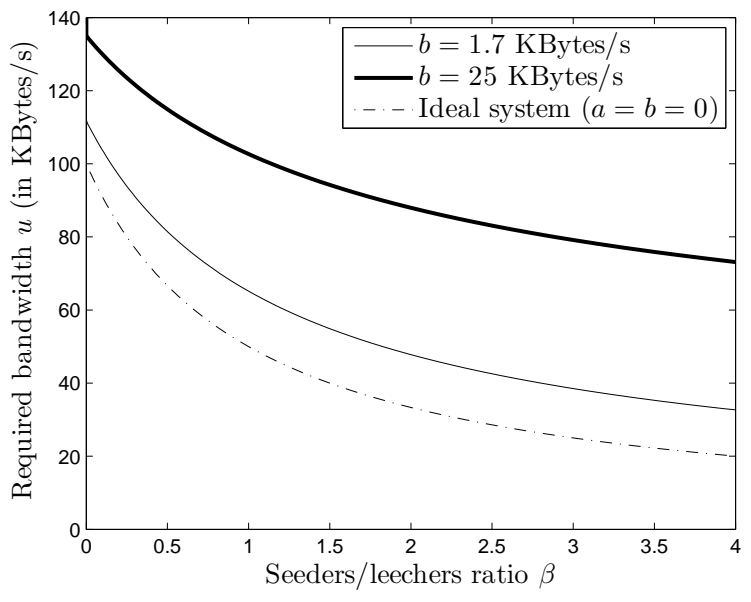

Fig. 6. Average bandwidth required for scalability

\section{Application: dimensioning a scalable live streaming system}

Many dimensioning rules can be derived by using the formulas we proposed. For instance, determining if the system is scalable would consist in checking if $\eta(L) \alpha_{L}+\eta(S) \beta \alpha_{S} \geq 1$ [2]. If we assume here for simplicity homogeneous bandwidth $u, \eta(S)=\eta_{O P T}(u)$ (neglecting aggregation issues), and optimal leechers' efficiency $\eta_{L}=\eta_{\max }{ }^{3}$, one can derive the relationship that $u$ and $\beta$ must verify for the system to be scalable:

$$
\beta \eta_{O P T}(u) \geq \frac{r}{u}-\eta_{\max } .
$$

If $\beta$, which indicates the ratio between idle (seeders) and active (leechers) users, is a given parameter of the system, Equation (26) can be used to derive the bandwidth $u$ that is required for the system to be scalable. This is illustrated by Figure 6 (the performance of the perfect system, i.e. $a=b=$ 0 , is also plotted for comparison). Notice how even little values of $\beta$ (less than 1) can give significant decrease of the required bandwidth, which is $R$ for a seedless system with perfectly efficient leechers.

\section{About delays}

We do not have taken delay issues into account. The diffusion delay is obviously a major concern in the design of a live streaming system. However, it should be noted that the two heuristics we proposed are based on diffusion trees. Therefore the induced delay is at most equal to the delay of a single connection times a logarithm of $N_{L}$. This is exactly the same type of delay that is experienced for diffusion based on leechers only, so we argue that using seeders should not impact the delay performance of a P2P live streaming system.

\footnotetext{
${ }^{3}$ The efficiency of leechers should take into account the number of outgoing connections like we did for the seeders. However, $\eta_{L}$ is not the main matter of this paper, so we assume without remorse perfect efficiency $\eta_{\max }$.
}

\section{CONCLUSION}

In this paper, we gave the keys to understand how seeders could be used in P2P live streaming if servers and leechers lack the necessary bandwidth. After a preliminary work on perfect and limited-fanout systems, we conducted our study on a model with linear overhead. The theoretical results presented in this preliminary study may have a significant impact in the design and dimensioning of live streaming systems using seeders.

In a future work, we plan to pursue the matter of leechers/seeders interaction in the general overhead model. We also think that the concept of live seeders introduced here could be extended to a more general concept of half-seeders, i.e. seeders with not all resources expected from a traditional seeder. Studying half-seeders could allow to extend our results to all P2P content distribution systems, including file-sharing and Video-on-Demand systems.

\section{ACKNOWLEDGMENT}

This work has been supported by the French Laboratory of Information, Network and Communication Science (LINCS).

\section{REFERENCES}

[1] B. Cohen, "Incentives Build Robustness in BitTorrent," in 1st Workshop on Economics of Peer-to-Peer Systems, 2003.

[2] F. Benbadis, F. Mathieu, N. Hegde, and D. Perino, "Playing with the bandwidth conservation law," in IEEE P2P, 2008. [Online]. Available: http://portal.acm.org/citation.cfm?id=1443451

[3] S. Liu, R. Zhang-Shen, W. Jiang, J. Rexford, and M. Chiang, "Performance bounds for peer-assisted live streaming," SIGMETRICS Perform., vol. 36, no. 1, pp. 313-324, 2008.

[4] T. Bonald, L. Massoulié, F. Mathieu, D. Perino, and A. Twigg, "Epidemic live streaming: optimal performance trade-offs," in SIGMETRICS '08: Proceedings of the 2008 ACM SIGMETRICS international conference on Measurement and modeling of computer systems. Annapolis, USA: ACM, 2008, pp. 325-336.

[5] D. Qiu and R. Srikant, "Modeling and performance analysis of bittorrent-like peer-to-peer networks," SIGCOMM Comput. Commun. Rev., vol. 34, no. 4, pp. 367-378, October 2004. [Online]. Available: http://dx.doi.org/10.1145/1030194.1015508

[6] D. Carra, G. Neglia, and P. Michiardi, "On the impact of greedy strategies in bittorrent networks: The case of bittyrant," in IEEE P2P, 2008, pp. 311-320. 